\title{
La responsabilidad social empresarial y el éxito competitivo de las micro, pequeñas y medianas empresas del cantón Jipijapa
} Corporate social responsibility and the competitive success of micro, small and medium-sized companies in the Jipijapa canton

\author{
MSc, Jaime Baque, Miguel Ángel ${ }^{1}$ \\ angel.m.86@hotmail.com \\ MSc, Baque Morán, Amparo Bienvenida ${ }^{1}$ \\ amparo.baque@unesum.edu.ec \\ MSc, Soledispa Rodríguez, Xavier Enrique ${ }^{1}$ \\ xsoledispa@hotmail.com \\ MSc, Cantos Figueroa Mariana de Lourdes ${ }^{1}$ \\ macafi1@yahoo.com \\ Merchán Conforme, Carlos Eduardo ${ }^{1}$ \\ eco.carlosmerchan@hotmail.com
}

Recibido: 1/12/2017, Aceptado: 1/02/2018

\begin{abstract}
RESUMEN
La objetividad y relevancia de esta investigación fue interpretar los factores que inciden en el cumplimiento de la responsabilidad social empresarial en el éxito competitivo de las pequeñas y medianas empresas, como eje prioritario en las funciones gerenciales. El trabajo investigativo enfoca la RSE en el ámbito de las mipymes del cantón Jipijapa. La metodología fue una investigación de campo, exploratoria, bibliográfica y de carácter descriptiva. Los resultados muestran que más de la mitad de las mipymes no están realizando actividades de responsabilidad social, relacionada con la calidad de vida de la empresa, el cuidado del medio ambiente, vinculación con la comunidad y el profesionalismo ético. Es decir, ninguna de las empresas cumple a cabalidad lo que es el término socialmente responsables, solo se limitan al trato e imagen ante el cliente. Desde este enfoque fue importante aportar con iniciativas innovadoras para los directivos en la efectiva toma de decisiones empresariales.
\end{abstract}

Palabras clave: Ética empresarial, mipymes, ambiente, innovación

$\overline{1 \text { Docente de la Universidad Estatal }}$ del Sur de Manabí. Ecuador

Revista científica Ciencia y Tecnología Vol 18 No 18 págs. 56-66 http://cienciaytecnologia.uteg.edu.ec 


\begin{abstract}
The Objectivity and relevance of this research was to interpret the factors that affect the compliance of corporate social responsibility in the competitive success of small and medium enterprises, as a priority axis in managerial functions. The research work focuses on CSR in the field of MSMEs in the Jipijapa canton. The methodology was a field, exploratory, bibliographical and descriptive research. The results show that more than half of the MSMEs are not carrying out activities of social responsibility, related to the quality of life of the company, the care of the environment, links with the community and ethical professionalism. In other words, none of the companies complies with what is the term socially responsible, they are limited to the treatment and image before the client. From this perspective, it was important to provide innovative initiatives for managers in effective business decision-making.
\end{abstract}

Keywords: Business ethics, SMEs, environment, innovation

\title{
Introducción
}

Desde años anteriores se menciona lo que es Responsabilidad, hoy en día se habla de responsabilidad social empresarial, que es un tema de relevancia, donde las empresas deben cumplir con el gran objetivo de ser responsable en su trato con los trabajadores, clientes, estado y con el medio ambiente. Desde este punto de vista las empresas deben participar con todos sus integrantes, con una variedad de ideas creativas e innovadoras que implementen sus estrategias empresariales.

Adoptar la responsabilidad social empresarial en el éxito de las pequeñas y medianas empresas, implica abrir el abanico emprendedor y a una nueva forma de gestionar, para mantener el foco en su estrategia de negocio y en el logro de la rentabilidad económica.

A partir de esto se asigna una relevancia mayor a los impactos de emprendimientos entre los diversos actores involucrados.

La RSE constituye pues una forma de manifestar la contribución de las empresas a la construcción de una sociedad mejor: más justa, más solidaria, más sostenible. La propia amplitud del concepto evidencia, por supuesto, que no existe un único modelo de responsabilidad social ni unas acciones específicas en las que deba forzosamente concretarse, pues cada empresa puede elegir muy diversos caminos de participación social y de contribución al bien común (Jáuregui, Martínez Pujalte, \& Torme, 2011) La RSE también se refiere al desarrollo de una nueva visión de la empresa como institución mucho más abierta a otras necesidades, intereses y expectativas diferentes a las de los propios gestores y accionistas, lo que exige la búsqueda de un equilibrio más equitativo entre los distintos grupos participantes en la actividad económica (Perdigueiro \& García, 2005) (Ibáñez \& Blanco, 2004). La RSE es el compromiso de las empresas con los valores éticos que dan un sentido humano a su empeño por el desarrollo sostenible, buscando una estrategia de negocios que integre 


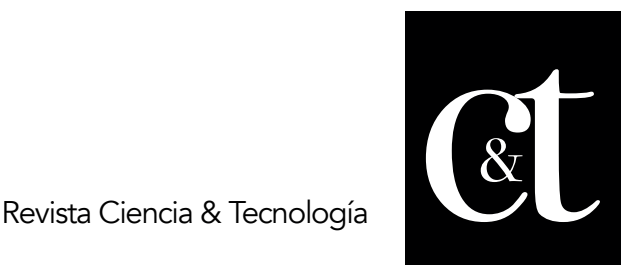

No. 18, 30 de abril de 2018

ISSN impreso: 1390 - 6321

el crecimiento económico con el bienestar social y la protección ambiental (Navarro, 2008).

Existen múltiples definiciones que tratan de acotar los márgenes de la responsabilidad social de la empresa. En nuestro ámbito geográfico más inmediato, la definición ofrecida por la Comisión Europea es, tal vez, la que ha tenido mayor éxito: "la integración voluntaria, por parte de las empresas, de las preocupaciones sociales y medioambientales en sus operaciones comerciales y en sus relaciones con sus interlocutores" (Comisión Europea, 2001, p.366). Por su parte, el Foro de Expertos define la RSE como aquella que, partiendo del cumplimiento de la ley, se basa en "la integración voluntaria en el gobierno de la empresa, en su gestión, su estrategia, sus políticas y sus procedimientos, de las preocupaciones sociales, laborales, medioambientales y de respeto a los derechos humanos que surgen de la relación y el diálogo transparentes con sus grupos de interés, responsabilizándose así de las consecuencias y los impactos que se derivan de sus acciones".

Una de las razones fundamentales para explicar el reciente auge de la RSE hay que buscarla en la incapacidad del mundo académico y empresarial para explicar las interrelaciones entre la empresa y su entorno social. Buena parte de esta deuda habría que imputarla a pautas de pensamiento tradicionales según las cuales toda empresa es, en esencia, un ente maximizador de beneficios que funciona con independencia del marco de relaciones e incentivos que se establecen con su entorno social y medioambiental más inmediato.

Lógicamente, con un aparato conceptual tan limitado, tan desligado de una realidad mucho más compleja, ha sido inevitable que, en el momento en que la sociedad, los gobiernos e, incluso, los mercados han empezado a demandar una visión mucho más plural de las organizaciones, la teoría económica tradicional se haya visto desplazada por lecturas mucho más sociológicas de lo que representa este entramado de relaciones entre individuos, organizaciones y gobiernos que forma lo que conocemos por economía para una aproximación, véase Biggart \& Beamish (2003).

La responsabilidad social empresarial y el emprendedor social

Investigaciones de Piñeiro \& Romero (2011), sobre la Responsabilidad Social Empresarial (RSE), fundamentan que el éxito empresarial no puede seguir sustentándose en bases tradicionales y afirman que las empresas, deben de asumir un proceso de renovación en sus estrategias de RSE y ofrecer respuestas renovadas a las necesidades de la sociedad dentro del marco sustentable y de apoyo a la misma. (Chirinos, Fernández, \& Sánchez, 2012), interpretan un mundo globalizado e interrelacionado, donde las grandes corporaciones multinacionales llegan a tener presupuestos muy altos y requieren normas de conducta empresarial que minimicen los impactos sociales y de medio ambiente en la actividad económica de dichas empresas. Catalin \& Carmen (2010) declaran que la aparición de lo social en el proceso de inversiones de las empresas, conducen a un nuevo concepto llamado inversiones socialmente responsables. 


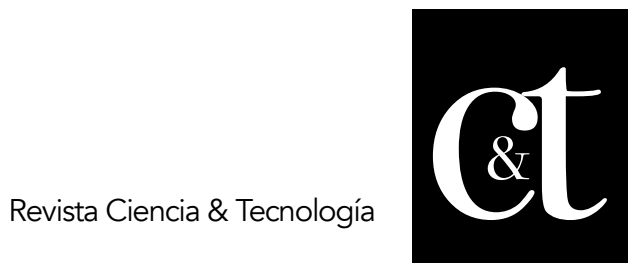

No. 18, 30 de abril de 2018

ISSN impreso: 1390 - 6321

Vives (2013) establece como parte de las estrategias de RSE ante la sociedad, el poder y deber de contribuir al empleo y el emprendimiento, a través de la creación de empleos directos, en condiciones compatibles con la dignidad humana y a la reducción del desempleo juvenil, a través de programas de aprendizaje y entrenamiento.

Silva (2007) establece que, en la visión global del mundo, surge el emprendedor social, con un espíritu solidario, creativo e intelectual, con una amplia preocupación social, voluntad política y actitud ética, con imaginación, capacidad y compromiso para crear valor agregado entre grupos de actores de la sociedad civil por el bien común.

Responsabilidad Social Empresarial, Innovación y Éxito competitivo de las Microempresas La relación entre la RSE y el valor empresarial ha sido objeto de varios estudios empíricos. Aunque autores como Margolis \& Walsh (2003), señalan que los distintos estudios que se vienen realizando en este tema han tenido resultados heterogéneos, mayoritariamente relaciones positivas (Pivato, Misani, \& Tencati, 2008), pero también hay autores que no consideran la relación suficientemente determinante como Blowfield \& Murray (2008). De hecho, Barnett (2007) indicaba que después de muchos años de investigación en esta línea no se puede determinar con rotundidad que la inversión en iniciativas sociales tenga un mayor retorno para los accionistas.

Sin embargo, hay áreas específicas en las que las acciones de RSE han sido reconocidas como verdaderos artífices de la mejora del rendimiento organizativo y esto es especialmente importante para las microempresas. Nos referimos a la mejora de la relación con los consumidores, la mayor facilidad para conseguir financiación, la atracción de talento o la protección de la reputación corporativa. Más concretamente, el éxito competitivo ha sido señalado como un logro clave tras la realización de acciones de RSE, entendiendo por éxito la obtención de unos resultados positivos para la empresa en términos de posicionamiento en el mercado y que van más allá del ámbito financiero (Fernández \& Santaló, 2010). Siendo esto así en las empresas en general, nuestra primera cuestión de investigación es: ¿Tiene la RSE alguna influencia en el éxito competitivo de las microempresas?

Por otro lado, autores como Surroca, Tribo, \& Waddock (2010), han señalado el efecto mediador de la innovación en el ámbito de la RSE. Ésta puede ser considerada en sí misma una innovación, pero, además, se plantea que las empresas que acometen acciones de RSE son más proclives a innovar. A su vez, la innovación aparece en la literatura como un factor clave para el éxito ya que son numerosas las investigaciones que han puesto de manifiesto la incidencia de la innovación en la generación de ventajas competitivas (Gibson \& Naquin, 2011).

La innovación no es un concepto nuevo, ya se aplicaba para aprovechar y crear riqueza dentro de las regiones, los países y las economías. La competitividad de las regiones en la economía cada vez más global ahora requiere no sólo que la innovación 




No. 18, 30 de abril de 2018

ISSN impreso: 1390 - 6321

esté presente dentro de una economía regional, necesita permear en mecanismos existentes para transferir eficazmente esas innovaciones desarrolladas desde el laboratorio de investigación, directamente al mercado. Porter, Bueno, Merino y Salmador (2010), padre de la estrategia competitiva moderna, introducen el concepto de innovación como ventaja competitiva dentro de la cadena de valor, conociendo las fuentes potenciales para crear ventajas sobre los competidores mediante el análisis de cada una de las actividades que se realizan en la empresa, diseño, producción, comercialización y distribución. La innovación tiene un papel de mediación entre la RSE y el éxito competitivo. La innovación tiende a aumentar cuando la empresa es socialmente responsable y el incremento de la innovación se traduzca en más éxito competitivo, potenciando el efecto que por sí misma ya ejercía la RSE en la competitividad de la entidad.

Por un lado, las innovaciones tecnológicas han sido señaladas como determinantes de la competitividad de las grandes empresas, aunque también las innovaciones organizativas, fundamentalmente en las empresas de menor tamaño, han sido destacadas por su capacidad para generar nuevos productos y servicios más competitivos (Perks \& Riihela, 2004).

\section{La gerencia y la Responsabilidad Social Empresarial}

Las empresas modernas no sólo tratan de vender los mejores productos al mejor precio, sino que también transmiten a la sociedad una determinada manera de hacer una cultura y unos valores éticos. En la gestión moderna de la empresa "no todo vale", la coherencia y las convicciones tienen que formar parte del producto que se ofrece (Jáuregui, Martínez-Pujalte, \& Torme, 2011).

El liderazgo de la alta dirección es uno de los componentes importantes del clima y cultura éticos pues el comportamiento de la gerencia es el factor más relevante para el desarrollo y mantenimiento de los dos; de ahí la importancia de que los gerentes comprendan y adopten el compromiso del comportamiento ético.

\section{Alcances de la Responsabilidad Social Empresarial}

En este escenario cambiante, donde aparece la Responsabilidad Social Empresarial como factor de equilibrio, se pueden visualizar los siguientes alcances como concepto en el desarrollo de las actividades empresariales:

- Alcance social y de desarrollo: Desde este punto de vista la RSE se centra en las actividades realizadas por la empresa con el fin de contribuir a la sociedad y la comunidad externa a ésta, cumpliendo con el sentido del deber y considerando en esto a los grupos más vulnerables.

- Alcance solidario e igualitario: La empresa se reconoce como un sistema inserto en uno mayor, destacándose ésta en la contribución a las oportunidades y la igualdad de las personas, en este sentido es que se reconoce la existencia implícita de derechos y deberes, por parte de las empresas y la sociedad.

- Alcance de autorreferencia: Aquí considera sólo el interior de la empresa, es decir, la relación que se produce entre ésta con los accionistas, clientes y trabajadores, sin que haya responsabilidades de otro tipo hacia la comunidad externa, enfocándose 


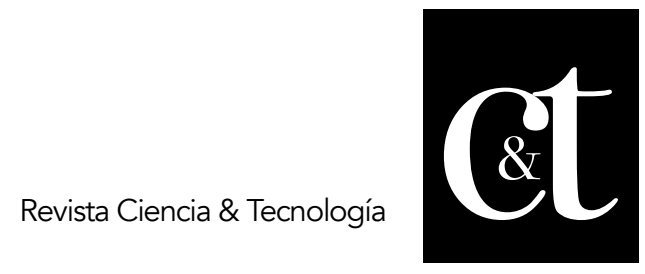

No. 18, 30 de abril de 2018

ISSN impreso: 1390 - 6321

solo al mercado y visualizando a la empresa como generadora de ganancias.

- Alcance ético sistémico: Se considera una visión integral de la empresa en la sociedad, donde se rescata la responsabilidad de ésta con sus stakeholders o grupos de interés de manera permanente, de ahí el carácter sistemático de esta noción.

- Alcance ético y de valores: Esta noción parte a raíz de lo expuesto en los puntos anteriores, considerando la RSE como reflejo de la empresa o las personas que están a cargo de éstas, destacando el énfasis de trabajar bajo valores y difundirlos a todas las áreas, fomentando el surgimiento de una ética corporativa que va a sustentar el proceso de toma de decisiones en la empresa.

\section{El camino hacia el éxito competitivo de la RSE}

Mientras que el desarrollo económico y social continúa mejorando las vidas de algunos en nuestro país, todavía hay trabajo considerable que hacer para alcanzar condiciones favorables en los ámbitos económico, social y ambiental que beneficien a toda la sociedad. Con la privatización aumentando a través de todo el hemisferio, la potencia y la influencia de las empresas continúan creciendo y es cada vez más evidente que la construcción de una sociedad más justa y una economía más sostenible depende, en gran parte, de influenciar a la comunidad de negocios para poner en ejecución acciones que permitan alcanzar estas metas. Los mismos indicadores, cuantitativos y cualitativos, servirán de base para que la empresa y sus directivos establezcan las medidas necesarias para hacer más efectiva y eficiente la manera en que la responsabilidad social se integra a sus estrategias medulares.

"La Responsabilidad Social Empresarial es hoy un valor agregado y una ventaja competitiva para la empresa. Aquellas empresas que desde hoy incorporen o refuercen sus acciones de responsabilidad social lo harán a su propio ritmo y de acuerdo a sus posibilidades; mañana tendrán la exigencia del entorno" (Villalobos, 2004).

"Es, al mismo tiempo, un imperativo para los negocios y una ventaja competitiva. Actualmente se considera que los pilares de una estrategia exitosa de negocios son: capital adecuado, buena gerencia, productos y servicios de calidad, dominio de la tecnología, servicio al cliente y una estrategia integral de responsabilidad social. Todos estos pilares están ligados entre sí y son interdependientes" (Cemefi y Empresa Socialmente Responsable, 2000).

Se puede indicar que esta investigación tuvo un fundamento muy relevante en base a otras investigaciones, las mismas que obtuvieron resultados impactantes por investigadores científicos reconocidos a nivel mundial, es decir toda responsabilidad social empresarial parte de ideas de negocios innovativos, creativos y con rigor ambiental, que sustentan la satisfacción de todas las partes interesadas.

"Cada vez más las empresas perciben que la responsabilidad social es un tema que no está restricto solamente a las acciones sociales o ambientales desarrolladas por la organización en la comunidad, sino que implica también las prácticas de diálogo e interacción con los diversos públicos de la empresa. Para que la empresa trabaje en 
el tema de la responsabilidad social, en una perspectiva sistémica y amplia, es necesario que éste sea incorporado a los procesos de gestión y, por lo tanto, tratarlo como parte de las estrategias de negocio y del sistema de planeación interna de la empresa" (Red Interamericana, 2005).

\section{Metodología}

La investigación fue de campo, exploratoria, bibliográfica y de tipo descriptivo con un enfoque cuantitativo, se realizó una descripción de las situaciones del contexto y de las circunstancias presentadas sobre la responsabilidad social empresarial y el éxito competitivo de las micro, pequeñas y medianas empresas del cantón Jipijapa, donde se observó detalladamente cómo funciona la intervención social que llevan Mipymes. El objetivo fue realizar una búsqueda específica de las características de su contribución social, en el cual se llegó a un análisis y conclusión de la problemática.

Mediante el estudio descriptivo se logró narrar la realidad de la intervención social que llevan a cabo las Mipymes, va directamente con el éxito competitivo, el mismo que presenta utilidad en la investigación y muestra con precisión la situación actual de las micro, pequeñas y medianas empresas del cantón Jipijapa en relación con el tema investigativo.

\section{Resultados}

Es trascendental, antes de presentar los resultados, caracterizar la muestra en base al tamaño micro, pequeñas y medianas empresas del cantón Jipijapa, permanencia en el mercado y escolaridad del empresario, para la efectiva interpretación de la información. Como escenario para el desarrollo de la investigación se tomó a 30 Mipymes existentes en la Zona Urbana del cantón Jipijapa, que estuvo divida en tres zonas:

Zona 1: 19 empresas ubicadas en la zona céntrica de la Ciudad de Jipijapa, que corresponde a la Parroquia urbana San Lorenzo.

Zona 2: 3 empresas ubicadas en la Parroquia Urbana Miguel Morán Lucio.

Zona 3: 8 empresas ubicadas en la parroquia Urbana Manuel Inocencio Parrales y Guale. Lo que resalta es que el estudio está conformado preponderantemente por micro, pequeñas y medianas empresas, dedicadas principalmente al sector comercial o de servicios y con empresas relativamente jóvenes.

Los resultados muestran que más de la mitad de las Mipymes no están realizando actividades de responsabilidad social. Lo que implica que ninguna cumple con la responsabilidad empresarial, solo se limitan al trato e imagen con el cliente. A continuación, se demuestra en forma específica los resultados de la encuesta.

\section{1. ¿Conoce usted el significado de Responsabilidad Social Empresarial?}

El 33\% respondió que sí conocía el significado y el $67 \%$ respondió que no conocía su significado. 
Se valida estos resultados de la encuesta, en su mayoría los propietarios de las Mipymes desconocen el significado de RSE. En consecuencia, es importante que se entienda este significado de Responsabilidad Social Empresarial, porque es importante conocer que los negocios están basados en principios ético y apegados a la ley. La empresa tiene un rol ante la sociedad, ante el entorno en el cual opera.

\section{2. ¿De qué manera usted asocia que las Mipymes tengan Responsabilidad Social} Empresarial?

Los encuestados manifestaron que el $40 \%$ menciono que retribuir a la sociedad por el consumo de su producto, el 33\% colaboración con la sociedad, el $20 \%$ oportunidad de promocionar las Mipymes y el $7 \%$ de actos de caridad.

A través de los resultados se puede deducir que el interés en mejorar aspectos sociales al momento de realizar acciones de RSE. Otro de los factores importantes es que algunos empresarios el término caridad lo consideran como una estrategia de publicidad, mientras que algunos dicen que no se puede tomar como una estrategia. Pero para las Mipymes debe ser comportarse éticamente y de contribuir al desarrollo económico de la comunidad local y la sociedad.

\section{3. ¿Cree usted qué su empresa tendría beneficios siendo una Empresa Socialmente Responsable?}

Se preguntó a los empresarios si sus empresas tendrían beneficios de RSE, el 17\% dijeron si y el $83 \%$ que lo desconocen totalmente, pero que de ser una empresa socialmente responsable les podría generar algunos beneficios.

Esto implica que los empresarios, no están siendo reconocido por alguna institución u organización que les que otorgue algún reconocimiento, premio y distintivos a las empresas que realizan prácticas de RSE para que de esta manera las firmas puedan contar con una ventaja de éxito competitivo.

4. ¿Qué acciones están realizando los empresarios de la Mipymes para beneficiar a sus empleados?

Los resultados de la encuesta a los empresarios de las Mipymes, sobre qué acción es realizan ellos para beneficiar a sus empleados, el $53 \%$ manifestó que es la convivencia, el $27 \%$ indicó que nada realizan a favor de sus empleados y el $20 \%$ manifestaron que les envían a tomar algunos cursos de capacitación y otorgan becas u apoyos para que sigan estudiando. Se nota que la mayoría de los empresarios indican que es fomentar la convivencia entre los empleados. Por lo tanto, es importante que los microempresarios ofrezcan mayores beneficios a sus trabajadores para generar a largo plazo una fuerza laboral de calidad y mejor capacitada.

5. ¿Qué incentivos otorgan las Mipymes cuando el trabajador aporta buenas ideas para mejorar las actividades de trabajo? 
Los empresarios de las Mipymes encuestados, manifestaron el $50 \%$ que ellos les entregan bonos a sus empleados, el $27 \%$ indicaron que nada, el $17 \%$ dice que les hacen un reconocimiento a sus empleados y el $7 \%$ les ascienden de puesto a su trabajo. Los resultados demuestran el tipo de incentivos que utilizan las Mipymes para premiar e incentivar los esfuerzos de sus trabajadores, cuando estos ellos aportan con buenas propuestas que les permitan a los empresarios mejorar en alguna área, siendo los bonos extra la forma más común de incentivarlos.

6. ¿Cree usted que las Mipymes logran el éxito competitivo teniendo una excelente responsabilidad social empresarial?

Los encuestados en un $83 \%$ indicaron que las mipymes logran su éxito competitivo teniendo una excelente responsabilidad social empresarial y el $17 \%$ manifestaron que no.

Cabe mencionar que los empresarios de las Mipymes están conscientes de ser responsables en el aspecto empresarial.

Otra de las preguntas que se realizó fue sobre:

7. ¿Cree que las Mipymes de esta ciudad están preocupadas por cuidar el medio ambiente?

La respuesta a esta pregunta, los empresarios consideran que las empresas muestran preocupación en cuidar el medioambiente; el $80 \%$ indican que si están preocupados en cuidar el medio ambiente y el $20 \%$ que no.

Uno de los criterios es que existe contaminación en los ríos y calles, que son provocadas por la misma ciudadanía, pero en la ciudad de Jipijapa no se tiene ninguna empresa grande que contamine el medio ambiente.

\section{Conclusiones}

La relevancia de esta investigación fue el cumplimiento de los objetivos, que era determinar los motivos por los que la empresa adopta la Responsabilidad Social Empresarial (RSE), analizar las principales acciones de RSE que implementan las Mipymes, así como las barreras que limitan dicho comportamiento. Debido a la poca importancia que se ha dado al estudio de la RSE en las Mipymes, por esta razón es prioritario realizar investigaciones que contribuyan a potenciar el nivel de conocimiento sobre responsabilidad social empresarial.

Cabe resaltar que se ha visto en investigaciones o estudios que las empresas están cada día acogiendo la filosofía de Responsabilidad Social Empresarial, aunque algunas las consideran como estrategias de RSE. Dichas acciones generalmente son esporádicas, pero aun así con las dificultades que existen para comportarse responsablemente, se tienen avances significativos sobre este tema. También se 
concluye que todo esto depende del grado de aceptación de la RSE en las Mipymes que depende exclusivamente del empresario. Por lo tanto, los esfuerzos deben enfocarse en las razones éticas del empresario para fomentar la RSE en las Mipymes.

Una de las limitaciones en este proceso investigativo está en la medición de la RSE en las Mipymes, pues se tienen elementos subjetivos y de percepción del empresario, por lo que es difícil evaluar las acciones directas de la RSE.

\section{Referencias bibliográficas}

Barnett, M. L. (2007). Stakeholder influence capacity and the variability of financial returns to corporate social responsibility. Academy of Management Review, núm. 32.

Biggart, N. W., \& Beamish, T. (2003). The economic sociology of conventions: habit, custom, practice and routine in market order. Annual Review of Sociology, 29: 443-464.

Blowfield, M., \& Murray, A. (2008). Corporate Social Responsibility. A Critical Introduction. Oxford University Press, Oxford.

Catalin, D. R., \& Carmen, C. N. (2010). The Growth of Organizations competitiveness though the Development of Social responsibility Investments Projects. Annals of the University of Oradea, Economic Science Series 17(4).

Cemefi y Empresa Socialmente Responsable (2000). III Congreso de Responsabilidad Social Empresarial en América. Fo. Fórum Empresa / Cemefi.

Chirinos, M. E., Fernández, L., \& Sánchez, G. (2012). Responsabilidad Empresarial o Empresas Socialmente Responsables. Razón y Palabra, número 81 noviembre 2012 - enero 2013.

Comisión Europea (2001). Promoting a European framework for Corporate Social Responsibility - Green Paper. Luxemburgo: Office for Official Publications of the. Europa: European Communities, COM.

Fernández, K. D. \& Santaló, J. (2010). When necessity becomes a virtue: The effect of product market competition on corporate social responsibility. Journal of Economics \& Management Strategy. Volume 19, Issue 2, Summer 2010.

Gibson, D. V., \& Naquin, H. (2011). Investing in innovation to enable global competitiveness: The case of Portugal. Technological Forecasting and Social Change. 78(8), 1299-1309.

Ibáñez, J., \& Blanco, F. (2004). Responsabilidad Social de la Empresa. Madrid: Ediciones Akal, S.A.

Jáuregui, R., Martínez-Pujalte, V., \& Torme, A. (2011). Responsabilidad Social Empresarial, Ideas y Reflexiones. Madrid España: Ceim.

Margolis, J. D., \& Walsh, J. P. (2003). Misery loves companies: Rethinking social initiatives by business, Administrative Science Quarterly. Universia Business Review, Vol. 48, núm. 2. 


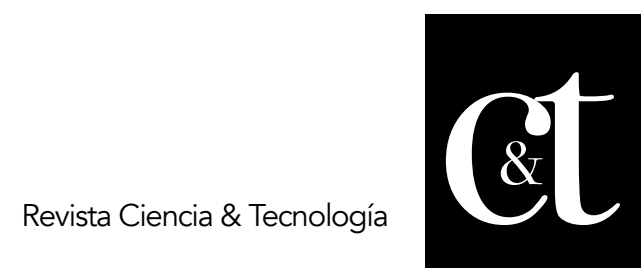

No. 18,30 de abril de 2018

ISSN impreso: 1390 - 6321

Navarro, F. (2008). Responsabilidad Social Corporativa: Teoría y Práctica. Madrid: Esic.

Perdigueiro, T., \& García, A. (2005). Crecimiento, Competitividad y Responsabilidad: Encrucijada Europea. En T. Perdigueiro \& A. García, La Responsabilidad Social de las Empresas y los nuevos Desafíos de la Gestión Empresarial. Barcelona: Universidad de Valencia.

Perks, H., \& Riihela, N. (2004). "An exploration of inter-functional integration in the new service development process". The Service Industries Journal, Vol. 24, núm. 6.

Piñeiro Chousa, J. y Romero Castro, N. (2011). Responsabilidad Social Empresarial y resiliencia. Revista Gallega de Economía, vol. 20, núm. 2, pp. 1-34.

Pivato, S., Misani, N. \& Tencati, A. (2008). The impact of corporate social responsibility on consumer trust: The case of organic food. Business Ethics $A$ European Review 17(1):3 - 12.

Porter, M., Bueno, E., Merino, C. y Salmador, M.P. (2010). Ventaja competitiva creación y sostenibilidad de un rendimiento superior. Madrid: Pirámide.

Red Interamericana (2005). Situación de la RSE en Latinoamérica hacia un desarrollo sustentable. RSE Interamericana CSR Network. Chile.

Silva, J. (2007). El emprendimiento social en el cambio de época. Worlds \& Knowledges Otherwise. Obtenido de http://www.documentacion.edex.es/docs/1704desemp.pdf

Surroca, J., Tribo, J., \& Waddock, S. (2010). Corporate Responsibility and Financial Performance: The Role of Intangible Resources. Strategic Management Journal 31(5), pp. 463-490.

Villalobos, J. (2004). Discurso Presidente Ejecutivo del Cemefi. II Conferencia Interamericana de Responsabilidad Social Empresarial. Del Dicho al Hecho, BID/Cemefi, México, D.F

Vives, A. (2013). Mirada crítica a la Responsabilidad Social de la empresa en Iberoamérica. Cumpetere. 